\title{
La cultura organizacional: el gran impulsor de la estrategia futura. Una visión de la competitividad en Ecuador
}

\section{Organizational Culture: the Great Driver of Future Strategy. A Vision of Competitiveness in Ecuador}

RECIBIDO: 10 DE MAYO DE 2020/ ACEPTADO: 2 DE JUNIO DE 2020

\section{ALbert COSTA MARCÉ \\ Universidad de Girona (España) \\ albert.costa@udg.edu}

\section{CARMEN ECHAZARRETA SOLER \\ Universidad de Girona (España) \\ carmen.echazarreta@udg.edu}

\section{Resumen}

Introducción: Ecuador está en la mitad inferior de la tabla de competitividad del World Economic Forum 2019. Metodología: A través de una revisión documental de los principales modelos y estudios relacionados, la presente investigación tiene como objetivo analizar las razones de la baja competitividad del modelo económico del Ecuador e identificar los factores determinantes para fomentar su recuperación y competitividad empresarial. Resultados: Más de una tercera parte de líderes y empresarios menciona que el tipo de liderazgo es transaccional o no tiene liderazgo; menos de la mitad poseen una creencia profunda; menos del 20\% posee un Consejo de Administración y el uso de tecnologías disruptivas es mínimo. Discusión: La cultura organizacional con centro en las personas, con códigos como la tecnología y el manejo de un buen gobierno corporativo, es indispensable para proyectar la empresa. Conclusiones: La competitividad está relacionada directamente con la capacidad de tener enfoque hacia el futuro.

Palabras clave: Cultura Organizacional; Competitividad; Liderazgo; Modelo de Negocio; Innovación.

\section{DIEGO IGNACIO MONTENEGRO GÁLVEZ \\ IDE Business School-Universidad de los Hemisferios (Ecuador) \\ diegom@uhemisferios.edu.ec}

\section{Abstract}

Introduction: Ecuador is in the lower half of the World Economic Forum's 2019 competitiveness table. Methodology: Through a documentary review of the main models and related studies, this research aims to analyze the reasons for the low competitiveness of Ecuador's economic model and identify the determining factors to promote its recovery and business competitiveness. Results: More than a third of leaders and entrepreneurs in the country mention that the type Leadership is transactional or has no leadership; less than half have a deep belief; less than $20 \%$ have a Board of Directors and the use of disruptive technologies is minimal. Discussion: The organizational culture with a focus on people, with codes such as technology and the management of good corporate governance, is essential to project the company. Conclusions: Competitiveness is directly related to the ability of companies to focus on the future.

Keywords: Organizational Culture; Competitiveness; Leadership; Business Model; Innovation. 


\section{INTRODUCCIÓN}

Pensar en el futuro es siempre una tarea compleja. Al ser humano le gus$\tan$ más los hechos que las suposiciones. Hunt asevera que "no sabemos lo que hacemos hasta que hacemos lo que no solemos hacer", pero hacer lo que no solemos hacer requiere un esfuerzo adicional para las personas y organizaciones porque se han acostumbrado a la rutina y se mantienen "prudentes" por el miedo. Y como dijo el mismo Hunt, "el miedo a lo desconocido promueve igual tipo de temor que tenían los antiguos marineros: si tienes miedo de caerte al final del mundo, tu pensamiento se mantiene cerca de la costa"1.

De manera general, las empresas, o cualquier organización, se acostumbran al presente, al terreno conocido, a la zona confortable. Parece ser que el futuro es la continuación del pasado; de las viejas prácticas gerenciales o de los antiguos paradigmas que funcionaron a su tiempo. Hugh Macleod menciona que "el cambio no es la muerte, el temor al cambio sî". Y en la actualidad, el cambio va a una velocidad mayor de la capacidad de adaptación de las personas. La aceleración de la Ley de Moore ${ }^{3}$ no le da tiempo a la humanidad para adaptarse al nuevo paradigma. Las organizaciones intentan entender las implicaciones de la transformación digital, cuando ya los científicos y futurólogos están hablando de una nueva era de transformaciones como la 5.0.

Además, Macleod afirma que "todo el mundo necesita esa idea loca, que está ahí fuera, que les permita de verdad empezar a hacer algo que le guste, algo que importe" 4 . Se requiere un plan que permita que las organizaciones sean más competitivas, alejándose de personas tóxicas y de los callejones sin salida que representa muchas veces el día a día.

En el desarrollo del presente artículo se dan pautas importantes para entender que las organizaciones de Ecuador han vivido en el día a día. Desde el tipo de liderazgo que se practica en las organizaciones; seguido por el tipo de enfoque, ya se basado en la eficiencia, las capacidades distintivas o la unidad de la organización; y, concluyendo en una visión a futuro y creencia profunda,

1 Hunt, J. (2010).

2 Macleod, H. (2011).

3 La Ley de Moore es un término informático originado en la década de 1960 y que establece que la velocidad del procesador o el poder de procesamiento total de las computadoras se duplica cada doce meses. En un principio, la norma no era muy popular pero sí se sigue utilizando hasta el día de hoy. Fue acuñada por Gordon Moore, el cofundador de la conocida empresa Intel de microprocesadores. https://www.vix.com/es/btg/curiosidades/4933/que-es-la-ley-de-moore-y-para-que-sirve consultada el $3 \mathrm{de}$ diciembre de 2019.

4 Macleod, H. (2011) 
con la necesidad de incorporar en la estrategia elementos relevantes como las tecnologías de la nueva era o formas de gobierno corporativo que impulsen el diseño de modelos estratégicos sostenibles. Entonces, lo primero que deben hacer las empresas de Ecuador, es tomar consciencia de la situación actual en estos factores descritos e iniciar un proceso de fortalecimiento; primero, de su cultura organizacional, para posteriormente tomar las elecciones correctas en el diseño de un modelo estratégico a futuro.

\section{OBJETIVOS}

El objetivo primario de esta investigación es analizar las razones de la baja competitividad del modelo empresarial de Ecuador y aportar una herramienta que ayude a la concientización de los elementos fundamentales que se deben considerar para fomentar la competitividad.

Adicionalmente, existen unos objetivos secundarios:

- Comparar a Ecuador con Chile, Colombia, Perú y España, para comprobar la posición de este país en los resultados de "dinamismo empresarial" en un contexto regional y cultural cercano.

- Establecer la forma de liderazgo y buen gobierno corporativo más utilizado en Ecuador, como elementos fundamentales dentro de un modelo de cultura organizacional que mejore la competitividad futura.

- Determinar en qué nivel se están usando las tecnologías de la era 4.0. en las organizaciones ecuatorianas, como un insumo más para construir mejoras en el diseño de la estrategia empresarial.

\section{METODOLOGÍA}

La presente investigación se lleva a cabo a través de una revisión documental de los principales modelos y estudios relacionados. Partiendo de datos que se mostrarán en los siguientes apartados, el presente estudio se enmarca en un tipo de investigación explicativa, porque busca la causa de por qué ocurren ciertos sucesos, y permite la comprensión sobre temas específicos y las posibles razones por las que sucede un fenómeno. Es un primer acercamiento al problema que se pretende estudiar y conocer.

Se trata entonces a través de datos, de tener la comprensión de un tema y distinguir las causas por las que ocurre un fenómeno durante el proceso de investigación para poder anticipar cambios. Esta investigación explicativa debe 
permitir más adelante replicar estudios para darles mayor profundidad y obtener puntos de vista sobre el fenómeno indagado.

En el estudio motivo de esta investigación, se quieren explicar las posibles causas del estado competitivo de Ecuador (y de sus organizaciones), que en la actualidad se encuentra en la parte inferior del ranking de competitividad en lo referente a "dinamismo empresarial". Para esto, el estudio se basa en un cuestionario en profundidad realizado por Montenegro, a finales del año 2019 en 276 empresas de Ecuador con ingresos de más de $\$ 5$ millones U.S.D. (dólares de Estados Unidos de Norteamérica), en diversas industrias y composición societaria.

\section{RESULTADOS}

En el Ecuador, país en la mitad de los dos hemisferios, la competitividad es un asunto por resolver. El World Economic Forum, sitúa a este país en el ranking 86 de 140 países investigados en diez pilares de competitividad, muy por debajo de otros países con los que comparte frontera como Colombia (puesto 60), Perú (puesto 63) o Chile que es el mejor ubicado de América Latina (posición 33). Los pilares que mide el World Economic Forum (WEForum) se muestran en la siguiente figura:
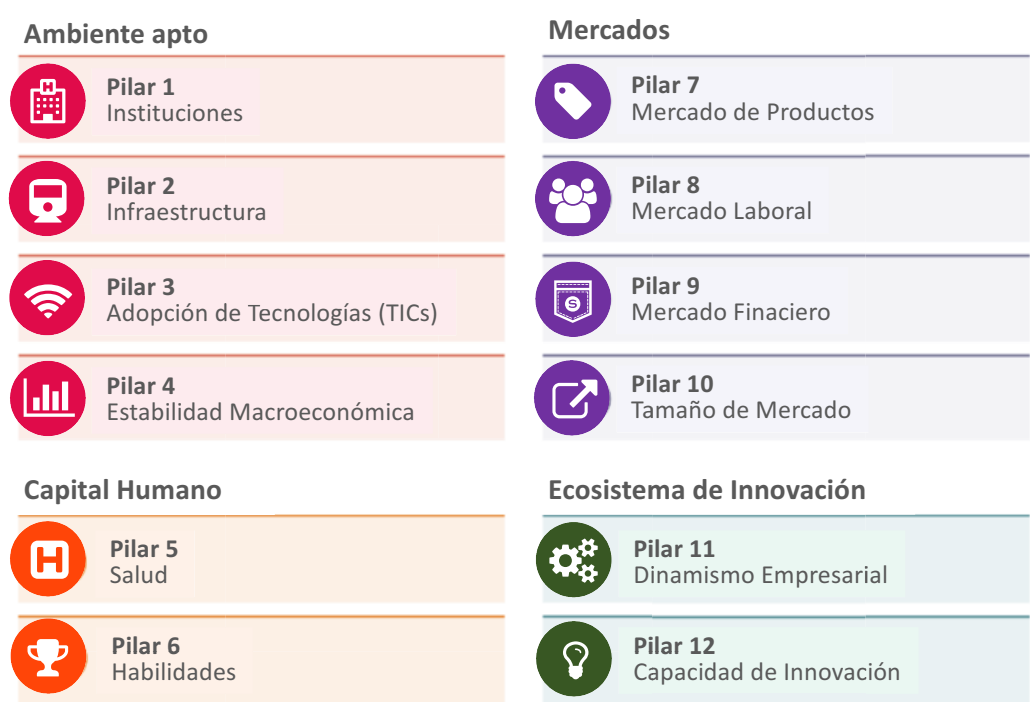

Imagen 1. Pilares del índice 4.o de Competitividad Global ( $\mathrm{GCl}$ 4.0.). Elaborado por Montenegro, D. I. Fuente: WEForum (2018). 
En el informe de 2018, el WEForum desarrolla un nuevo índice que denomina "4.0.", que añade a los pilares de ambiente apto (lo básico para la competitividad), capital humano y mercados, el análisis del "ecosistema de innovación” debido a la aceleración de las tecnologías que impactan directamente en la innovación.

En el pilar "dinamismo empresarial" el Ecuador tiene uno de los puntajes más bajos del ranking (129 de 140 países investigados en 2018) con una calificación de 45,2 sobre 100 puntos. El mejor puntaje en este pilar lo tiene Estados Unidos. El WEForum define "dinamismo empresarial" como: "la capacidad de las empresas del sector privado para adoptar nuevas tecnologías y formas de gestionar el trabajo a través de una cultura organizacional que considera el cambio, el riesgo, los nuevos modelos estratégicos y las reglas de negocio que permiten a las empresas ingresar y salir del mercado con facilidad. Un sector ágil y dinámico aumenta la productividad al asumir riesgos comerciales, probar nuevas ideas y, como consecuencia, diseñar soluciones innovadoras para las organizaciones. En un sector caracterizado por una redefinición permanente, los sistemas económicos exitosos son resistentes a los choques tecnológicos y pueden reinventarse a sí mismos”. Para 2019, el WEForum (2019) coloca a Ecuador en el ranking 90 países y en el pilar 11 "dinamismo empresarial" el puesto 130 de 141 países investigados.

Walsh afirma que las organizaciones deben ir hacia el futuro y regresar desde el futuro al presente para llevar a cabo lo que han visionado en ese posible futuro. Este proceso, como se mencionó en la introducción, no es tarea fácil, porque las organizaciones tienen un "sistema inmunológico" que les impide "ver más allá de lo evidente" y regresar al status quo; tratan de explicar su comportamiento con base en hechos pasados y, en las actuales circunstancias, el futuro ya no es la continuación del pasado ${ }^{5}$.

En el presente, la organización tiene, generalmente, una mentalidad de "arreglar y mejorar"; de tratar de explotar al máximo las capacidades para conseguir los resultados que se ha propuesto en el corto plazo. Por tanto, la empresa trata de generar objetivos y tareas para cumplir esos objetivos operativos con plazo de un año. Importan los resultados mensuales, el día a día, los silos departamentales o áreas de trabajo y las recompensas monetarias. Pérez-López menciona que: "La actividad real de una persona en una organización viene determinada tan sólo parcialmente por el sistema operativo compuesto

5 Walsh, M. (2019). 
de dos vertientes: la actividad requerida y la actividad espontánea" ${ }^{\text {. Se en- }}$ tiende por "sistema operativo", desde la concepción de la tecnología al programa o software básico de un ordenador que facilita la interacción entre el usuario y los demás programas del ordenador, y también con el hardware o dispositivos físicos. La cultura organizacional viene siendo como el sistema operativo que une la creencia, valores y personas de la empresa con la estrategia que, al final, es el camino para conseguir los resultados. Sin ese "sistema operativo" nada en la organización funcionaría correctamente.

Actualmente, la cultura organizacional es limitada (o casi inexistente), porque se "ahoga" en la operación de explotación de corto alcance de la empresa. Es muy probable, entonces, que la empresa se deshumanice. Andreu y Rosanas $^{7}$ proponen siete pilares para humanizar a la empresa: (1) concebir la empresa como lo que es, una comunidad de personas; (2) servicio de la persona; (3) volcarse en el aprendizaje y desarrollo personal; (4) pedir una mayor rendición de cuentas de los directivos de la empresa; (5) adoptar una visión holística que respeta la diversidad; (6) reequilibrar los sistemas de retribución; y, (7) aspirar a una misión más elevada.

Sobre este último punto es importante detenerse. Aspirar a una misión más elevada es mirar hacia futuro. El WEForum al hablar del pilar "dinamismo empresarial" menciona que el trabajo empresarial se debe gestionar a través de una cultura organizacional que considera el cambio, el riesgo, los nuevos modelos estratégicos y las reglas de negocio. Si no se mira el cambio y el riesgo como necesario, no se podría tener una misión elevada y un futuro competitivo. A continuación, se muestra la variación de los indicadores de cultura empresarial dentro del pilar de dinamismo empresarial de Ecuador frente a algunos países de la región y España (por la cercanía cultural con Ecuador):

6 Pérez-López, J. A. (1993).

7 Andreu, R. y Rosanas, J. M. (2012). 


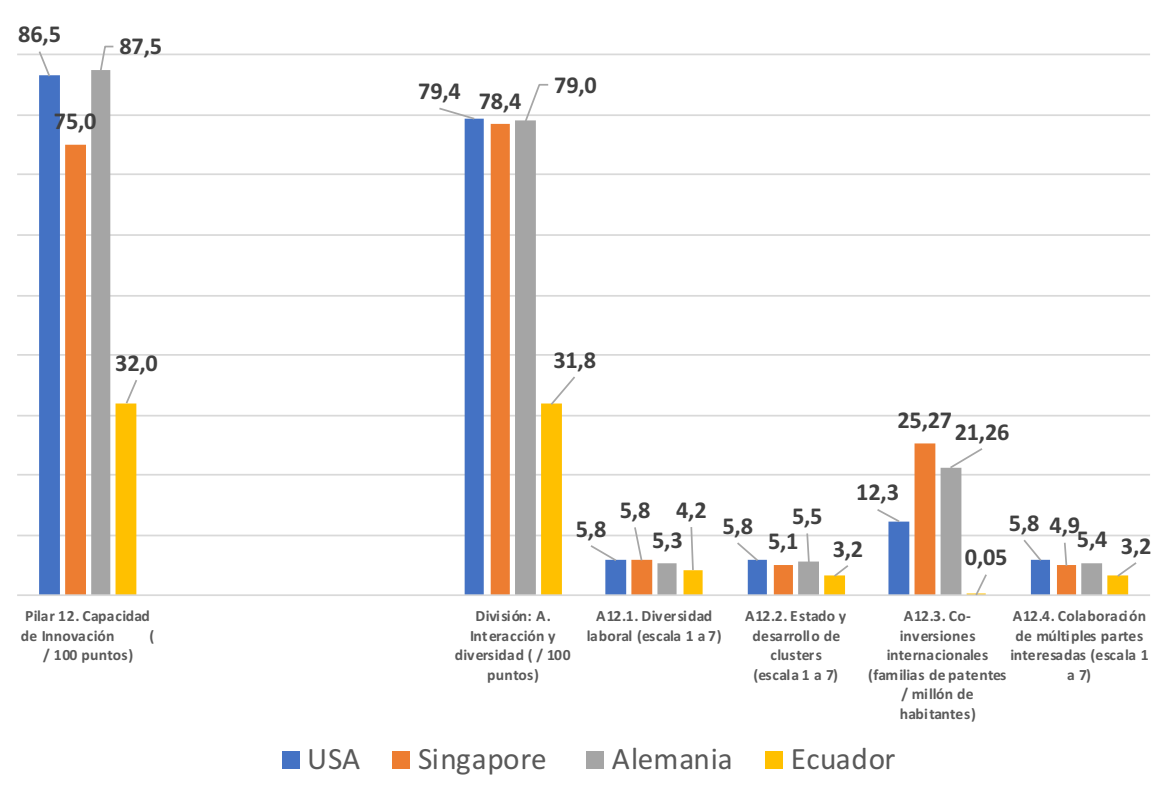

Gráfico 1. Puntajes de indicadores del pilar 11. Dinamismo empresarial, división B. Cultura Empresarial del GCl 4.o., comparando Ecuador con Chile y países fronterizos, se incluye a España.

Fuente: WEForum (2018).

En el anterior gráfico se puede apreciar la calificación sobre 100 puntos de cada uno de los países investigados en el pilar 11 "dinamismo empresarial". Por ejemplo, Ecuador tiene un resultado de 45,2 sobre 100 puntos en este pilar, mientras que España tiene 66,3. Adicionalmente, se muestra el resultado específico de cada componente de la "cultura empresarial" dentro del pilar "dinamismo empresarial"; así, Ecuador tiene una valoración de 3,4 sobre 7 puntos en el componente "crecimiento de empresas innovadoras", mientras que España 3,9. 


\begin{tabular}{|l|c|c|c|c|}
\hline $\begin{array}{l}\text { Categoría: Ecosistema } \\
\text { de Innovación. }\end{array}$ & $\begin{array}{c}\text { Variación } \\
\text { Ecuador vs. } \\
\text { España (\%) }\end{array}$ & $\begin{array}{c}\text { Variación } \\
\text { Ecuador vs. } \\
\text { Chile (\%) }\end{array}$ & $\begin{array}{c}\text { Variación } \\
\text { Ecuador vs. } \\
\text { Colombia (\%) }\end{array}$ & $\begin{array}{c}\text { Variación } \\
\text { Ecuador vs. } \\
\text { Perú (\%) }\end{array}$ \\
\hline $\begin{array}{l}\text { Pilar 11. Dinamismo } \\
\text { Empresarial. }\end{array}$ & $-31,8 \%$ & $-29,7 \%$ & $-28,9 \%$ & $-17,1 \%$ \\
\hline $\begin{array}{l}\text { División: B. Cultura } \\
\text { Empresarial. }\end{array}$ & $-9,0 \%$ & $-15,4 \%$ & $-8,4 \%$ & $-3,0 \%$ \\
\hline $\begin{array}{l}\text { B11.1. Actitud hacia el } \\
\text { riesgo empresarial. }\end{array}$ & $2,6 \%$ & $-7,1 \%$ & $5,4 \%$ & $-2,5 \%$ \\
\hline $\begin{array}{l}\text { B11.2. Disposición para } \\
\text { delegar la autoridad. }\end{array}$ & $-11,4 \%$ & $-29,1 \%$ & $-9,3 \%$ & $-2,5 \%$ \\
\hline $\begin{array}{l}\text { B11.3. Crecimiento de } \\
\text { empresas innovadoras. }\end{array}$ & $-12,8 \%$ & $-15,0 \%$ & $-8,1 \%$ & $-2,9 \%$ \\
\hline $\begin{array}{l}\text { B11.4. Empresas que } \\
\text { adoptan ideas disruptivas. }\end{array}$ & $-3,1 \%$ & $-8,8 \%$ & $-8,8 \%$ & $3,3 \%$ \\
\hline
\end{tabular}

Tabla 1. Variación porcentual indicadores del pilar 11. Dinamismo empresarial, división B. Cultura Empresarial, comparando Ecuador con los países de la región y España.

Fuente: WEForum (2018).

En la tabla precedente, se puede notar la diferencia porcentual entre Ecuador y cada uno de los países del estudio del World Economic Forum. Por ejemplo, Ecuador en el pilar 11 "dinamismo empresarial" tiene una brecha de $29,7 \%$ frente a Chile y $31,8 \%$ con España calculado sobre la base de la calificación mostrada en el gráfico 1. En el componente "crecimiento de empresas innovadoras", dentro de cultura empresarial, Ecuador tiene una diferencia negativa del $15 \%$ frente a Chile y $12,8 \%$ con España.

Heffernan menciona que "la cultura de empresa, que está más allá de lo mensurable y a veces parece ir también más allá de la comprensión, se ha convertido en el ingrediente secreto de la vida de las organizaciones, aquello que marca la diferencia, pero para lo cual nadie tiene una receta" ${ }^{8}$. A pesar de que no existe una fórmula clara, la cultura empresarial está compuesta de pequeñas acciones, hábitos, elecciones e hipótesis. Estos comportamientos constituyen el principal impulsor para llevar a la organización a futuro; la empresa viene siendo como la bala que se coloca dentro de un cañón (que representa a la cultura organizacional); y, que la impulsa hacia una mentalidad de creci-

8 Heffernan, M. (2017). 
miento y capacidad de exploración más allá de la explotación de los recursos actuales. Este futuro (que puede ser más o menos incierto) ya no es la continuación del pasado; ya no se persigue un crecimiento lineal sino un crecimiento más radical en un trabajo más interconectado con otras empresas y personas, y con un nuevo flujo de conocimiento que no necesariamente es el formal. Una representación gráfica de lo descrito se muestra en la siguiente figura:

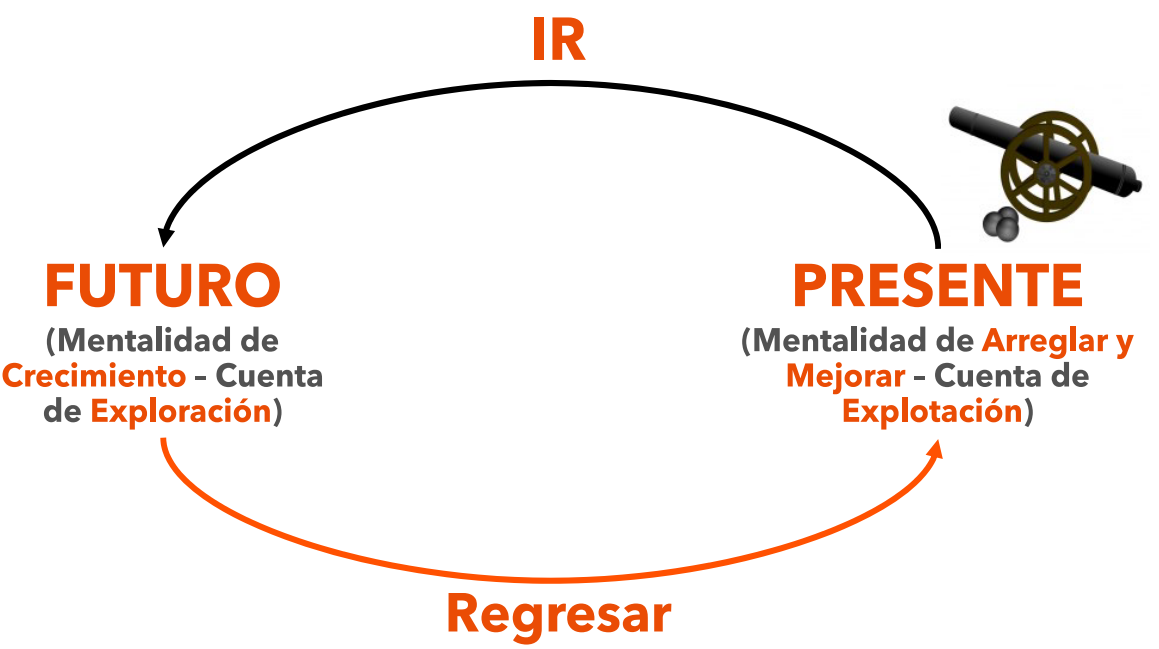

Imagen 2. Representación gráfica de la cultura organizacional como impulsora para llevar a la empresa del presente al futuro.

Fuente: Montenegro, D. I. (2019).

"La cultura de las organizaciones está compuesta por valores, creencias y normas. Los valores se refieren a la importancia que la compañía otorga a los aspectos de calidad, servicio al cliente y trato a los trabajadores. Las creencias son las ideas que las personas dentro de una organización mantienen respecto a ellos mismos y la empresa. Por último, las normas son las reglas no escritas que guían la forma de interactuar y el comportamiento de las personas"9. Pero, creencias y normas, ampliando el concepto de Flamholz, tienen una base muy fuerte en el significado de la palabra liderazgo; que constituye, dentro de esta

9 Flamholtz, E. G. (1995). 
perspectiva, el primer gran ingrediente de la "nueva receta" de una cultura organizacional.

Según Cardona, la teoria de liderazgo mas aceptada en los ultimos años es la que distingue entre liderazgo transaccional y transformador. El líder transaccional se apoya en los premios y castigos para motivar a sus subordinados. Su estilo directivo es de mando y control, con fuerte uso del poder formal. Es un líder que mira con detalle el corto plazo y que maneja los procesos y los recursos de manera eficiente. El líder transformador, en cambio, se caracteriza por tener una vision radicalmente nueva, que es atrayente y motiva a la gente. Es un líder inconformista, visionario y carismatico, que transforma tanto el estado de las cosas en la empresa como las mismas aspiraciones e ideales de los seguidores.

Manuel Barroso también establece una relación importante entre conciencia y trascendencia al decir que: "Se vive para elevar la conciencia a niveles superiores, para desprenderse de mapas y comportamientos inútiles, para descubrir el significado de lo que se es uno mismo y de lo que se hace, y trascender e ir más allá de lo material. La conciencia es la única herramienta para alinearse con el universo, para recuperar la ecología personal y social, y fortalecer la conexión con todo lo que está alrededor" ${ }^{\prime 10}$. Kofman afirma que el líder debe ejercer un "liderazgo consciente"; esto implica que debe liderar considerando los siguientes actitudes e interacciones: responsabilidad incondicional, integridad esencial, humildad ontológica, comunicación auténtica, negociación constructiva, coordinación impecable y, como consecuencia de lo anterior, conseguir maestría emocional. Además, promueve y demanda que sus colaboradores posean y pongan en práctica esas cualidades ${ }^{11}$.

En una investigación realizada en Ecuador por Montenegro se obtuvieron datos sobre una base de 276 empresas con más de $\$ 5$ millones de ingresos en ese país. Las preguntas fueron contestadas por líderes o gerentes de primera línea. Los resultados, cuando se les preguntó ¿qué tipo de liderazgo tiene la empresa?, fueron los siguientes:

10 Barroso, M. (2014).

11 Kofman, F. (2008). 


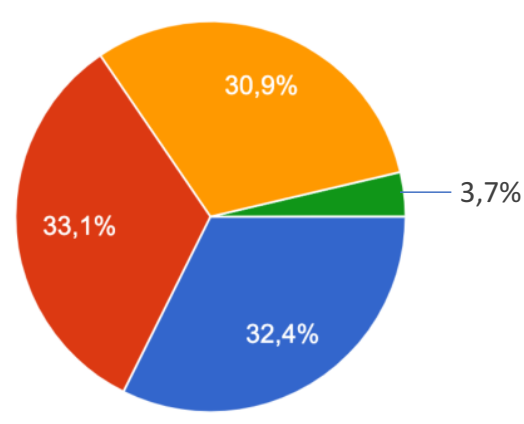

Liderazgo Transaccional: premios y

castigos para motivar a los empleados.

Liderazgo Tranformador: visionario y carismático que transforma las aspiraciones e ideales de las personas.

Liderazgo Trascendente: con una misión clara de servicio para los demás.

No existe liderazgo dentro la organización.

Gráfico 2. Resultados de la pregunta: ¿Qué tipo de liderazgo se ejerce en la organización? Investigación de competitividad en la era 4.0., en Ecuador.

Fuente: Montenegro, D. I. (2019).

Las respuestas estuvieron casi divididas en tercios iguales. El 36,1\% ejerce un liderazgo transaccional de corto plazo o no tiene liderazgo. Un $33,1 \%$ basa su liderazgo en la figura de un líder visionario y carismático, es decir, en una figura emblemática a la que las personas siguen. El menor resultado se obtuvo en el "liderazgo trascendente", directamente relacionado con una consciencia de servicio a otros sin mediar nada a cambio.

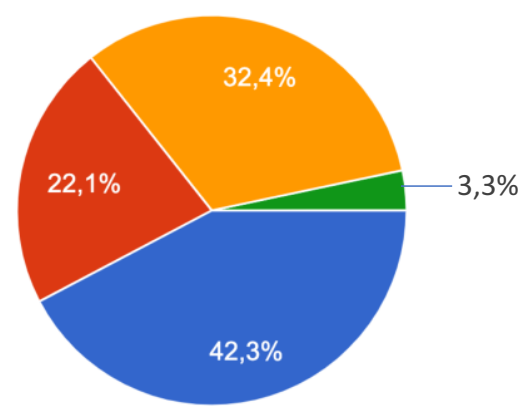

La eficacia, es decir a los resultados

económicos y los recursos empleados.

Al desarrollo de capacidades

distintivas de los colaboradores para

que resuelvan mejor los problemas.

A generar confianza y compromiso

entre colaboradores y con la

organización.

Ninguna de las anteriores

Gráfico 3. Resultados de la pregunta: ¿Considera que el líder se enfoca más en? Investigación de competitividad en la era 4.0., en Ecuador.

Fuente: Montenegro, D. I. (2019). 
La respuesta más significativa fue la de eficacia con el $42,3 \%$. Por lo tanto, a pesar de que, en la pregunta de tipo de liderazgo las respuestas fueron en tercios iguales, en esta interrogante predomina en una mayor proporción la búsqueda de resultados económicos y el uso de recursos en el corto plazo.

De cualquier forma, el liderazgo debe tener la capacidad para transformar la visión futura en estrategia. Esta visión, cobijada por una creencia profunda, es el segundo ingrediente de la cultura organizacional. Barraza ${ }^{12}$ indica que "visión de una empresa" describe sus planes, el mundo, sus clientes y a sí misma. Es decir, muestra cómo le gustaría que el mundo fuese dentro de algunos años. Esto incluye una idea acerca de qué cambios quieren ver en el mundo. También acerca de cómo se imaginan que la vida de sus clientes mejora. Y por último describe qué tipo de organización les gustaría ser, aunque esto es lo menos importante de la visión. Como tal, la visión de una empresa indica a dónde desea llegar la misma a largo plazo. Simon Sinek pone en el centro del modelo las creencias; los líderes inspiradores comienzan identificando su propósito, creencia, causa o visión... ${ }^{13}$.

Macleod subraya que "para capear las nuevas realidades hay que ser más creativo...no sólo dentro de la profesión particular, sino en todo. La manera de ver el mundo tendrá que hacerse más fértil y original. Y esto no es sólo así para los artistas, escritores, técnicos, directores creativos y consejeros delegados; es así para todos. También para los conserjes, recepcionistas y conductores de autobús. Las viejas maneras han muerto. Y se necesita de gente alrededor que coincida con uno" 14 .

Por tanto, la creencia profunda tiene que ser virtuosa y enfocada en personas (el centro del modelo cultural son las personas); la tecnología es un importante driver ${ }^{15}$ que mueve la innovación en las empresas, pero las personas son la razón de ser de una organización sustentada en una creencia. "Tener un valor es estar en buena forma interior, comportarnos de acuerdo con la dignidad que tenemos como personas, y en ese sentido ser estimados, ser apreciados como tales, como fruto de lo que somos"16.

12 Barraza, H. J. (2019).

13 Sinek, S. (2009).

14 Macleod, H. (2009).

15 Significado de driver: motor o impulsor clave del crecimiento, en https://www.linguee.es/ingles-espanol/traduccion/driver.html consultado el 5 de diciembre de 2019.

16 Yarce, J. (2009). 
En Ecuador (respuestas de la misma investigación realizada por Montenegro), el 80,9\% de empresas contestaron tener una visión, 79\% una misión; y, apenas un $42,6 \%$ mencionaron mantener una creencia profunda. ${ }^{17}$ Echazarreta y Costa en su estudio del "Valómetro" consideran que "en la visión, la empresa se plantea lograr retos de futuro comprometidos con el desarrollo de las personas y la sostenibilidad del medio" ${ }^{18}$. Parece la definición más adecuada, porque coloca a las personas como protagonistas de esa visión. El indicador que proponen Echazarreta y Costa para medir la visión es el porcentaje de coincidencia entre los retos de futuro de la empresa en comparación con los retos que definen los Objetivos de Desarrollo Sostenible (ODS) de Naciones Unidas que se indican a continuación:

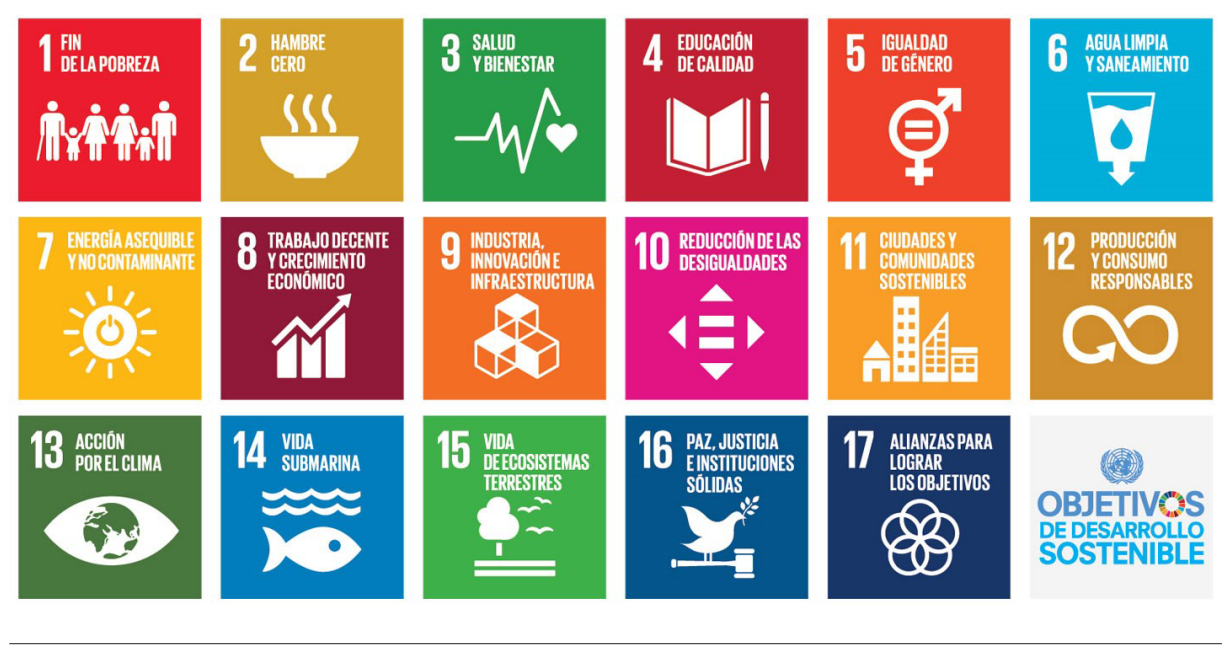

Imagen 3. Objetivos de Desarrollo Sostenible (ODS)

Fuente: Naciones Unidas (2015).

En Ecuador, de acuerdo con la investigación realizada por Montenegro en 2019, el mayor enfoque de las 276 organizaciones encuestadas está en el ODS "trabajo decente y crecimiento económico con un 61,4\% de las empresas encuestadas, seguido del ODS "producción y consumo responsable con un $33,8 \%$ y "salud y bienestar" con un $32 \%$. Los demás ODDs tienen resul-

17 Montenegro, D. I. (2019).

18 Echazarreta, C. A. y Costa, A. (2019). 
tados menores o nos son considerados de manera relevante por las organizaciones ecuatorianas entrevistadas ${ }^{19}$.

\section{DISCUSIÓN}

Según Jim Collins, los líderes tienden a tener egos monumentales que no pueden subyugar algo más grande y más sostenible que ellos mismos, es decir, sus empresas. Pero para los líderes que buscan la trascendencia, las condiciones adecuadas, como la autorreflexión o un evento profundamente transformador, como una enfermedad potencialmente mortal, pueden estimular la germinación del liderazgo humilde. Collins argumenta que el ingrediente clave que permite que una empresa se convierta en grande, es tener un líder de Nivel 5: "Un ejecutivo en el que la humildad personal genuina se mezcle con una intensa voluntad profesional" 20 .

"La humildad personal genuina" se consigue cuando se piensa en hacer el bien a otras personas, cuando todos se preocupan por el bienestar y crecimiento de otros. Las empresas deben cuidar, entonces, que su equipo corporativo sea un "buen gobierno corporativo" que cuide la creencia, la estrategia para llegar al largo plazo y los beneficios (en ese orden). Un buen gobierno corporativo es un tercer elemento de la cultura organizacional y resulta evidente que la responsabilidad de proyectar la empresa a largo plazo y conseguir, además, resultados a corto plazo, es del consejo de administración (conocido también como "directorio" o "board"), y del comité de dirección ("la administración" o "gerencia"). Sin embargo, no existe un tratamiento suficientemente refinado de las funciones de estos órganos de gobierno. La experiencia de cualquier empresa es que el trabajo de estos órganos de gobierno es crítico para el éxito de la organización.

Muy particularmente, la coordinación de estos órganos resulta imprescindible para lograr un adecuado sistema de gobierno de la empresa, de modo que los solapes de atribuciones y funciones, y asumir las responsabilidades que cada uno tiene, no entorpezcan la buena marcha de la empresa ${ }^{21}$. Como se está hablando de un modelo presente-futuro, dentro del diseño de la estrategia el buen gobierno corporativo, también se tiene que velar por la proyección ha-

19 Montenegro, D. I. (2019).

20 Collins, J. (2005).

21 Canals, J. (2004). 
cia ese futuro que sugiere algo diferente: "la empresa debe utilizar los mecanismos de gobierno corporativo para evitar poner en peligro su continuidad, pero también debe impulsar su crecimiento, su innovación, su inversión, sin las cuales no cabe supervivencia a largo plazo"22. Apenas un 10,3\% de las organizaciones investigadas en Ecuador afirman contar con un directorio o consejo de administración con miembros independientes, un $9,8 \%$ dice tener un protocolo de familia, $18,5 \%$ menciona que no tiene gobierno corporativo de ningún tipo ${ }^{23}$.

Por último, es inexcusable que una organización que quiere proyectarse hacia el futuro no disponga de una "caja de herramientas" que la vayan preparando para todos los cambios y riesgos a asumir. En este artículo se va a mencionar el driver o código cultural llamado "tecnología"; existen otros drivers, pero la tecnología, como dice Hunt, "es cada día más fuerte, pero su trompeta no siempre preludia la llegada de la caballería. Algunas de sus notas pueden ser muy agudas y, extrañamente, en ningún otro lugar este es más obvio que en el muy específico sector de la creación de algo nuevo. Por supuesto que nada ha cambiado tanto la existencia de las personas como el chip integrado. Internet une a todos como un pegamento. Las comunicaciones son un depredador que está muy por encima de las nubes siempre listo para cazar. Pero a pesar de todo lo que se recibe, a veces recibe demasiado"24.

Friedman señala que el ejemplo más esclarecedor sobre el avance de la tecnología lo mencionó en una entrevista para él, el consejero delegado de laboratorio Google X Eric "Astro" Teller. Google X o simplemente "X" es un laboratorio de investigación y desarrollo que, entre otras innovaciones, ha fabricado el coche sin conductor de Google. El punto, explicó Teller, ilustra un hecho importante: "a pesar de que los seres humanos y las sociedades se han adaptado paulatinamente a los cambios, de promedio la velocidad del cambio tecnológico está acelerándose tanto que ha subido por encima de la velocidad promedio a la que la mayoría de las personas pueden asimilar todos esos cambios. Muchos de nosotros no podemos seguir el ritmo, y esto está causando una ansiedad cultural que también nos está impidiendo beneficiarnos totalmente de toda la tecnología"25.

22 Canals, J. (2000).

23 Montenegro, D. I. (2019).

24 Hunt, J. (2010).

25 Friedmann, T. L. (2018). 
Pero, está claro que va a ser muy difícil que disminuya la aceleración de las tecnologías que muchas veces no comprendemos. La velocidad es fundamental en la estrategia de esta era. Kasparov ${ }^{26}$, el famoso campeón de ajedrez señaló que ya en 1987 disputó un torneo de "ajedrez rápido". Fue el primer campeonato serio de este tipo, con un ritmo de juego realmente acelerado. En aquellas partidas rápidas, disponíamos únicamente de veinticinco minutos cada uno para hacer todos nuestros movimientos, algo realmente muy distinto del ajedrez tradicional, cuyas partidas llegan a durar siete horas. El viejo paradigma ha cambiado tan radicalmente que Ismail, Malone y Van Geest ${ }^{27}$ afirman que se habla de "organizaciones exponenciales", cuyo impacto es desproporcionalmente grande (al menos 10 veces más grande), comparado con otras empresas y que se debe al uso de tecnologías exponenciales. Gutsch ${ }^{28}$ señala que el mundo está en caos y cambio y, por lo tanto, se debe ser más innovador, mejor administrador y buen inversor.

El mundo resuelve sus problemas complejos con un nuevo algoritmo. Desde técnicas que permiten imitar la inteligencia humana, pasando por formas en las que las máquinas mejoran las tereas aprendiendo y terminando en el aprendizaje de las máquinas imitando al cerebro humano, todo está cambiando a gran velocidad ${ }^{29}$. La creación de valor no se consigue solamente mejorando la eficiencia. Se necesita crecer en desempeño y en velocidad si se quiere tener un futuro. La aceleración producto de la tecnología es notoria y en cualquier campo. Walsh ${ }^{30}$ nos ofrece algunos ejemplos de tecnología y nuevas formas de gestión que van a cambiar el antiguo paradigma:

Biomecatrónica: es una ciencia interdisciplinaria que integra la biología, la neurociencia, la mecánica, la electrónica y la robótica, para reparar e incrementar las capacidades del cuerpo humano.

Blockchain: es una gran base de datos encriptada con el expediente público de todas las transacciones de Bitcoin que alguna vez han sido ejecutadas.

Cloud (la nube): es la red de servidores que en conjunto ofrece la informática y el software como un servicio y no como un producto.

Gamification: es la integración de la lógica del juego dentro de las actividades de negocio como el marketing o la motivación de personas.

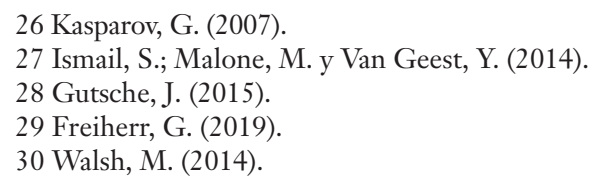


Holacracy: es un sistema de auto-gobierno de los colaboradores, en donde tienen diferentes roles conectados, circulares y no tienen un área de responsabilidad específica o nivel de reporte.

Inteligencia artificial: es la capacidad de una máquina de emular cualidades humanas como la percepción, patrones de reconocimiento y juicio.

Big Data: es un conjunto de datos suficientemente grande que desafía los enfoques convencionales de gestión y análisis.

Realidad virtual: es un conjunto de tecnologías de inmersión, capaces de crear la impresión de estar dentro de un entorno construido digitalmente.

Pharmacogenomics: es un campo emergente de la tecnología que analiza como genética individual responde al uso de los fármacos.

Internet de las cosas (IoT): es una red emergente de objetos conectados, capaz de sentir su entorno y compartir información.

Walsh describe 88 conceptos (que él llama ideas peligrosas) que pueden cambiar el futuro (o el presente) para siempre ${ }^{31}$. Cuando se les preguntó a las empresas del Ecuador sobre el uso de estas tecnologías o conceptos para el desarrollo de su estrategia futura, los resultados de no utilización de estas tecnologías fueron los siguientes ${ }^{32}$ :

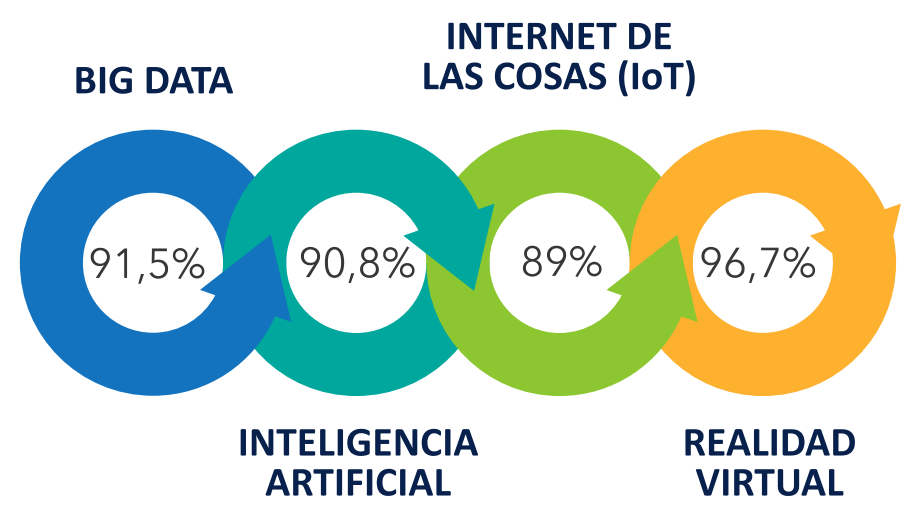

Gráfico 4. Resultados de no utilización de tecnologías. Investigación de competitividad en la era 4.o., en Ecuador. Fuente: Montenegro, D. I. (2019).

31 Porcentaje de organizaciones que no usan el concepto o la tecnología o lo usan de manera limitada. 32 Montenegro, D. I. (2019). 
Echazarreta y Costa incluso proponen indicadores de medición para las nuevas tecnologías exponenciales, como los que se indican a continuación:

\begin{tabular}{|c|c|c|}
\hline \multicolumn{3}{|l|}{ SECCIÓN E: TECNOLOGÍA } \\
\hline CRITERIOS & $\begin{array}{l}\text { No DE REFERENCIA } \\
\text { DEL INDICADOR }\end{array}$ & $\begin{array}{l}\text { INDICADORES } \\
\text { BÁSICOS }\end{array}$ \\
\hline $\begin{array}{l}\text { E8. INTELIGENCIA ARTIFICIAL. } \\
\text { Área multidisciplinar que intenta } \\
\text { dotar a las máquinas de procesos } \\
\text { cognitivos típicos del razonamiento } \\
\text { humano como deducir, razonar y } \\
\text { resolver problemas complejos. In- } \\
\text { cluye la rama de machine learningo } \\
\text { aprendizaje automático, que pre- } \\
\text { tende desarrollar técnicas que per- } \\
\text { mitan a las máquinas aprender au- } \\
\text { tomáticamente. }\end{array}$ & E8.1 & $\begin{array}{l}\text { Recursos económi- } \\
\text { cos anuales de la } \\
\text { empresa destinados } \\
\text { a la inteligencia artifi- } \\
\text { cial en euros. }\end{array}$ \\
\hline $\begin{array}{l}\text { E9. TECNOLOGíA INMERSIVA. } \\
\text { La Realidad aumentada (RA) es la } \\
\text { tecnología que ofrece información } \\
\text { digital superpuesta a la visión real. } \\
\text { La realidad virtual o simulación (RV) } \\
\text { se utiliza en fábrica para recrear un } \\
\text { espejo del mundo físico en un mo- } \\
\text { delo virtual. La realidad mixta (RM) } \\
\text { es la combinación de realidad virtual } \\
\text { y realidad aumentada. }\end{array}$ & E9.1 & $\begin{array}{l}\text { Recursos económicos } \\
\text { anuales de la em- } \\
\text { presa destinados a la } \\
\text { tecnología inmersiva } \\
\text { en euros. }\end{array}$ \\
\hline
\end{tabular}

Tabla 2. Muestra de Indicadores del "Valómetro" que tienen relación con Tecnología.

Fuente: Echazarreta, C. A. y Costa, A. (2019).

Las tecnologías exponenciales no están todavía en la mente de los empresarios ecuatorianos, o no existe una claridad de los conceptos y su aplicación para los modelos de negocio o la estrategia. Las aplicaciones se limitan a el uso de la nube, seguridad lógica y ciberseguridad.

Aspirar a mantenerse competitivo es un patrimonio exclusivo de empresas que son capaces de visionar ese futuro y ejecutar en el presente lo que sea 
necesario para crear valor incremental. Esto se llama enfoque. Las organizaciones (y personas) líderes deberían poner toda su determinación, persistencia y migrar el conocimiento para poder impactar hacia delante y ser sostenible en el tiempo. Esto requiere de un cambio de paradigma (un nuevo algoritmo) para el diseño del modelo estratégico alineado a esa visión y creencia.

Luego, con las elecciones adecuadas se construyen las hipótesis necesarias para poder responder al desafío (los problemas que se tienen que resolver y las oportunidades que se pueden aprovechar mejor que otros). El modelo se ejecuta con proyectos, iniciativas y tareas que llevan a la realidad esas hipótesis. Es un ejercicio de mirar hacia delante, pero también controlar la operación de la organización.

El impulsor necesario para que esto suceda es el sistema operativo llamado cultura organizacional. Es el cañón que envía con fuerza a la empresa hacia lo que "quiere" conseguir y no hacia lo que "puede" lograr. Nada funciona sin sistema operativo y nada alcanza aceleración sin una fuerza impulsora. Los elementos de la cultura empresarial explicados y propuestos en el presente artículo se muestran consolidados en la siguiente figura: 


\section{CULTURA HUMANISTA}

(Sistema operativo para la estrategia)
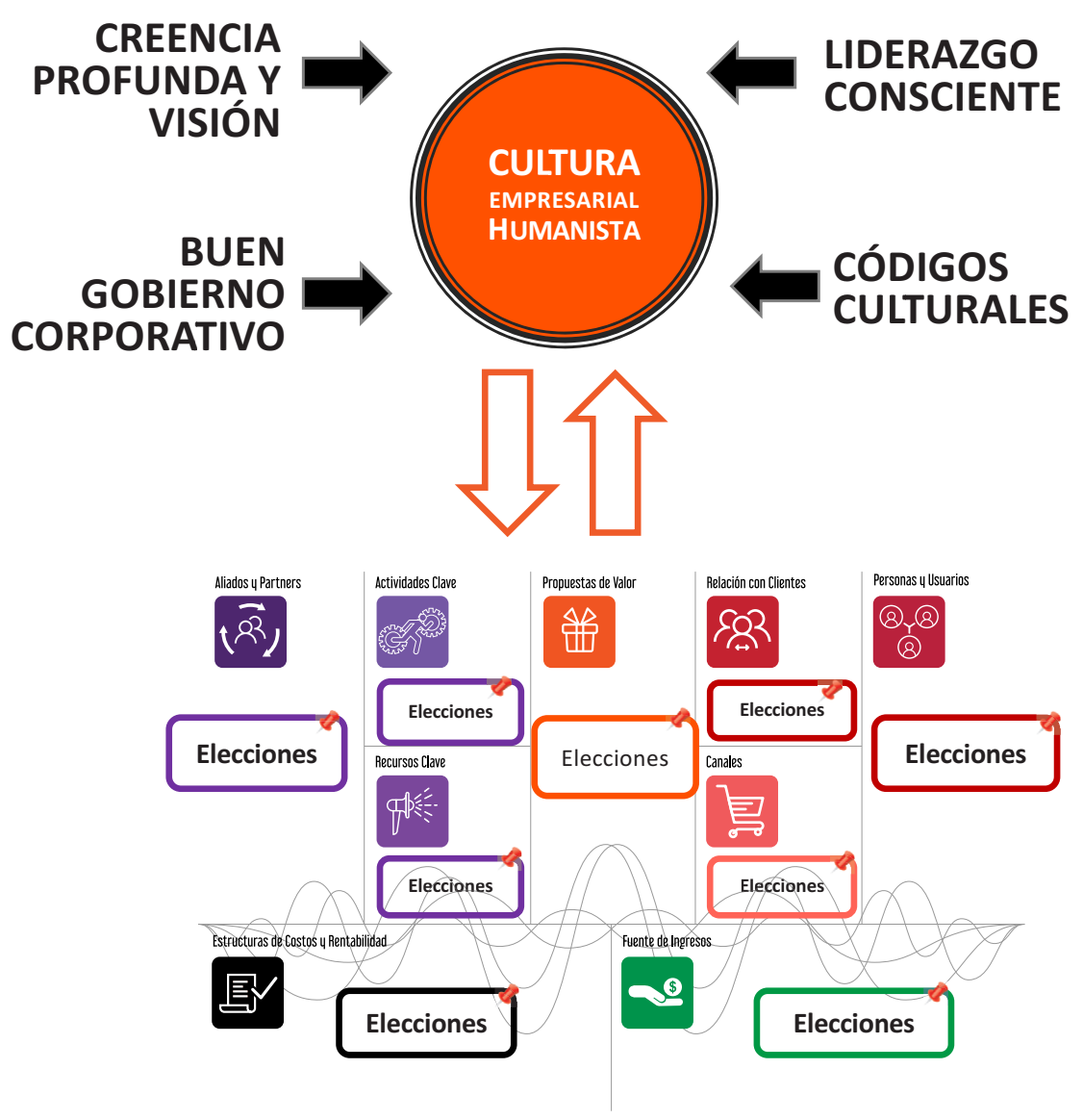

\section{MODELO ESTRATÉGICO}

(Con elecciones de hipótesis y supuestos futuros)

Imagen 4. Elementos de una cultura empresarial humanista (centro en la persona) y despliegue en un modelo estratégico con elecciones para el futuro.

Fuente: Montenegro, D. I. (2019). 


\section{CONCLUSIONES}

Como aporte para entender la baja competitividad de las organizaciones de Ecuador, se incluye en este estudio una herramienta de visualización y alineación de los elementos de una cultura empresarial humanista (liderazgo consciente, creencia profunda y visión, tecnología como driver o código cultural y buen gobierno corporativo) con el modelo estratégico. Sin cultura empresarial es poco probable que las elecciones que se realizan en la estrategia sean sostenibles en el tiempo y generen valor diferenciador.

Comparando los componentes de la cultura empresarial de Ecuador con los países de la región y España, Ecuador tiene desventajas en todos estos elementos (actitud hacia el riesgo, disposición a delegar la autoridad, crecimiento de empresas innovadoras y adopción de ideas disruptivas), excepto en la comparación frente a Perú en el componente "adopción de ideas disruptivas". Esto nos puede llevar a la conclusión de que los modelos para la competitividad adoptados por otros países cercanos pueden tener mejores resultados o a que, definitivamente, el modelo ecuatoriano es deficiente. Miño considera que el éxito del modelo chileno, por ejemplo, se debe a la liberación del mercado que ha presenciado desde la década de los setenta; el ingreso per cápita de Chile se ha cuadruplicado desde 1975, lo que lo convierte en el país más próspero de América Latina ${ }^{33}$. El 78\% de las empresas en Chile son de propiedad familiar y generan el $60 \%$ de las ventas en diversos sectores de la producción. La capacidad de generar modelos estratégicos basados en liderazgo, gobernanza, educación de las personas y en su visión a largo plazo, es parte del éxito de este país.

La cultura de las organizaciones es parte del pilar "dinamismo empresarial", donde Ecuador mantiene brechas negativas entre 17,1\% y $31,8 \%$ con Perú, Colombia, Chile y España, dejándolo en la parte inferior del ranking de competitividad mundial (ubicación 130 de 141 países en 2019).

La mayoría de las empresas entrevistadas en Ecuador señala que tienen un liderazgo transaccional en lugar de otro tipo de liderazgo más consciente o trascendente. También existe un porcentaje de 3,7\% que afirman que no existe liderazgo en la organización. Esto tiene relación con el enfoque a la eficiencia que el $42,3 \%$ de las empresas ecuatorianas mantienen como propuesta de valor genérica. La sostenibilidad de esta visión corta se ve complicada aún más

33 Miño, J. (2019). 
por la ausencia de un gobierno corporativo que soporte una visión independiente y estratégica del negocio. Apenas un 10,3\% de las empresas en Ecuador afirman contar con un consejo de administración o directorio con miembros independientes y menos el 10\% de las empresas familiares cuentan con un protocolo de actuación.

La adopción de tecnologías de la nueva generación también es una tarea pendiente en las elecciones estratégicas para conseguir un modelo competitivo en las empresas de Ecuador. Solamente el $8 \%$ en promedio, tienen de alguna manera incorporada a su estrategia y confían en tecnologías como la ciencia de los datos, la inteligencia artificial, el internet de las cosas o la realidad virtual. Según un estudio de OBS Business School, ya en el año 2015 el $33 \%$ de las empresas colombianas confiaban en que la ciencia de los datos le iba a permitir generar ventajas competitivas ${ }^{34}$. Colombia supera a Ecuador en todos los indicadores referentes a cultura empresarial.

Este artículo es una aproximación a las causas más visibles de la falta de competitividad en las organizaciones de Ecuador y es insumo fundamental para el diseño de metodologías y herramientas para lograr modelos estratégicos sostenibles, ágiles y que respondan de mejor manera a eventos imprevistos que afecten la economía y el bienestar de las personas.

34 OBS Business School (2017). 


\section{REFERENCIAS}

Andreu, Rafael \& Rosanas, Josep María (2012), Manifiesto por una Gestión Empresarial más Humana, IESEInsight, pp. 13-19.

Barraza, H. J. (26 de enero de 2019), “¿Cuál es la diferencia entre Propósito, Misión y Visión?”, Revista Entrepreneur en https://www.entrepreneur.com/article/294059

Barroso, Manuel (2014), Conciencia: Significado y Trascendencia, CreateSpace Independent Publishing, Madrid.

Canals, Jordi (2000), Managing Corporate Growth, Oxford University Press, Oxford.

Canals, Jordi (2004), Consejos de Administración y Buen Gobierno, IESE Publishing, Barcelona.

Cardona, Pablo (2001), Liderazgo Relacional, IESE Publishing, Barcelona.

Collins, Jim (2005), "Level 5 Leadership: The Triumph of Humility and Fierce Resolve", Harvard Business Review, vol. 1, pp. 1-12.

Echazarreta, Carmen A. y Costa, Albert (2019), "Estudio y Conceptualización del 'Valómetro': un Sistema de Indicadores Empresariales para la Gestión basada en Valores”, Revista Latina de Comunicación Social, vol. 74, pp. 573593.

Flamholtz, Eric G. (1995), "Managing Organizational Transitions: Implication for Corporate and Human Resource Management", European Management fournal, vol. 13, $\mathrm{n}^{\circ}$ 1, pp. 39-51.

Freiherr, Greg (2019), "Imaging Technology News", AI algorithm Detects Breast Cancer in MR Images, https://www.itnonline.com/article/ai-algorithmdetects-breast-cancer-mr-images-0 (Recuperado en noviembre de 2019).

Friedman, Thomas L. (2018), Gracias por llegar tarde, Grupo Planeta, Barcelona.

Gutsche, Jeremy (2015), Better and Faster, Penguin Random House, New York.

Heffernan, Margaret (2017), Más allá de lo medible, Urano, Barcelona.

Hunt, John (2010), El arte de la idea y cómo puede cambiar su vida, Urano, Barcelona. 
Ismail, Salim; Malone, Michael S. \& Van Geest, Yuri (2014), Exponential Organizations, Diversion Books, New York.

Kasparov, Gari (2007), Cómo la vida imita al ajedrez, Debate, Buenos Aires.

Kofman, Fredy (2008), La Empresa Consciente, Aguilar, Buenos Aires.

Macleod, Hugh (2009), Ignora a todos, Urano, Barcelona.

Macleod, Hugh (2011), Planes diabólicos, Urano, Barcelona.

Miño, José (2019), "El modelo económico de Chile es un caso de éxito en una Sudamérica propensa a la crisis", Mises Institute, https://mises.org/es /wire/el-modelo-económico-de-chile-es-un-caso-de-éxito-en-una-sudamérica-propensa-la-crisis, (Recuperado en mayo de 2020).

Montenegro, Diego I. (2019), Investigación de Competitividad de la Empresa ecuatoriana en la era 4.0. Pichincha, Quito.

OBS Business School (2017), Estudio OBS: Big Data en números, https://obsbusiness.school/es/noticias/estudio-obs/estudio-obs-big-data-ennumeros, (Recuperado en mayo de 2020).

Perez-López, Juan Antonio (1993), Fundamentos de la Dirección de Empresas, Rialp, Madrid.

Sinek, Simon (2009), Start with Why, Portfolio, New York.

Walsh, Mike (2014), The Dictionary of Dangerous Ideas, Lucy HowardTaylor, Hong Kong.

Walsh, Mike (2019), The Algorithmic Leader, Amanda Lewis, Vancouver.

World Economic Forum (2018), The Global Competitivenes Report 2018, en http://reports.weforum.org/global-competitiveness-report-2018/

World Economic Forum (2019), The Global Competitiveness Report 2019, en http://www3.weforum.org/docs/WEF_TheGlobalCompetitivenessReport2019.pdf

Yarce, Jorge (2009), El Poder de los Valores, Universidad de La Sabana. 